\title{
Advancement in research and therapy of NF1 mutant malignant tumors
}

\author{
Junyan Tao, Dantong Sun, Lina Dong, Hua Zhu* and Helei Hou* (D)
}

\begin{abstract}
The NF1 gene encodes neurofibromin, which is one of the primary negative regulatory factors of the Ras protein. Neurofibromin stimulates the GTPase activity of Ras to convert it from an active GTP-bound form to its inactive GDPbound form through its GTPase activating protein-related domain (GRD). Therefore, neurofibromin serves as a shutdown signal for all vertebrate RAS GTPases. NF1 mutations cause a resultant decrease in neurofibromin expression, which has been detected in many human malignancies, including NSCLC, breast cancer and so on. NF1 mutations are associated with the underlying mechanisms of treatment resistance discovered in multiple malignancies. This paper reviews the possible mechanisms of NF1 mutation-induced therapeutic resistance to chemotherapy, endocrine therapy and targeted therapy in malignancies. Then, we further discuss advancements in targeted therapy for NF1-mutated malignant tumors. In addition, therapies targeting the downstream molecules of NF1 might be potential novel strategies for the treatment of advanced malignancies.
\end{abstract}

Keywords: NF1, Ras, Therapeutic resistance, Molecular mechanism, Malignancies

\section{Background}

With the aging population and changes in lifestyle, the morbidity and mortality rates of malignancies in the world are rising drastically. In 2018, 18.1 million new cases and 9.6 million cancer deaths were registered worldwide [1]. Treatments for advanced malignant tumors, including traditional treatment (surgery combined with radiotherapy and chemotherapy), targeted therapy and immunotherapy, significantly prolong the survival of patients with malignancies. To date, targeted therapies have achieved significant advances. The studies on the driven mutations of malignancies, such as epidermal growth factor receptor (EGFR), anaplastic lymphoma kinase $(A L K)$, ROS proto-oncogene 1 (ROS1) and human epidermal growth factor receptor-2 (HER2), have brought clinical benefits and more therapeutic options for patients with malignancies [2-4]. Patients harboring

*Correspondence: 1272668226@qq.com; houhelei@qdu.edu.cn Precision Medicine Center of Oncology, the Affiliated Hospital of Qingdao University, No. 59 Haier Road, Qingdao, Shandong 266000, China sensitive mutations benefit from targeted therapy. However, drug resistance remains a serious problem during treatment. Drug resistance limits the use of targeted therapy in malignant tumors and is one of the foremost challenges in malignant tumors today [5].

Neurofibromin 1 (NF1) mutations cause an autosomal dominant genetic susceptibility syndrome known as neurofibromatosis type 1 [6]. Furthermore, genomic data from the cBioPortal for Cancer Genomics datasets indicate that somatic NF1 mutations can be detected in a variety of malignancies, including non-small-cell lung cancer (NSCLC), ovarian cancer, breast cancer, liver cancer, and esophagogastric cancer. The alterations and frequencies of NF1 in malignancies are shown in Fig. 1. According to previous studies, NF1 mutations have been detected in patients with primary and acquired resistance to tyrosine kinase inhibitors (TKIs) [7, 8]. In this case, we conclude the latest advancement in targeted therapy of malignant tumors with NF 1 mutations and further clarify the role of NF1 mutations in the treatment of malignancies to establish the viability of the treatment targeting NF1 mutations.

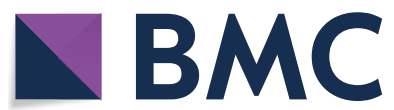

(c) The Author(s) 2020. This article is licensed under a Creative Commons Attribution 4.0 International License, which permits use, sharing, adaptation, distribution and reproduction in any medium or format, as long as you give appropriate credit to the original author(s) and the source, provide a link to the Creative Commons licence, and indicate if changes were made. The images or other third party material in this article are included in the article's Creative Commons licence, unless indicated otherwise in a credit line to the material. If material is not included in the article's Creative Commons licence and your intended use is not permitted by statutory regulation or exceeds the permitted use, you will need to obtain permission directly from the copyright holder. To view a copy of this licence, visit http://creativeco mmons.org/licenses/by/4.0/. The Creative Commons Public Domain Dedication waiver (http://creativecommons.org/publicdomain/ zero/1.0/) applies to the data made available in this article, unless otherwise stated in a credit line to the data. 

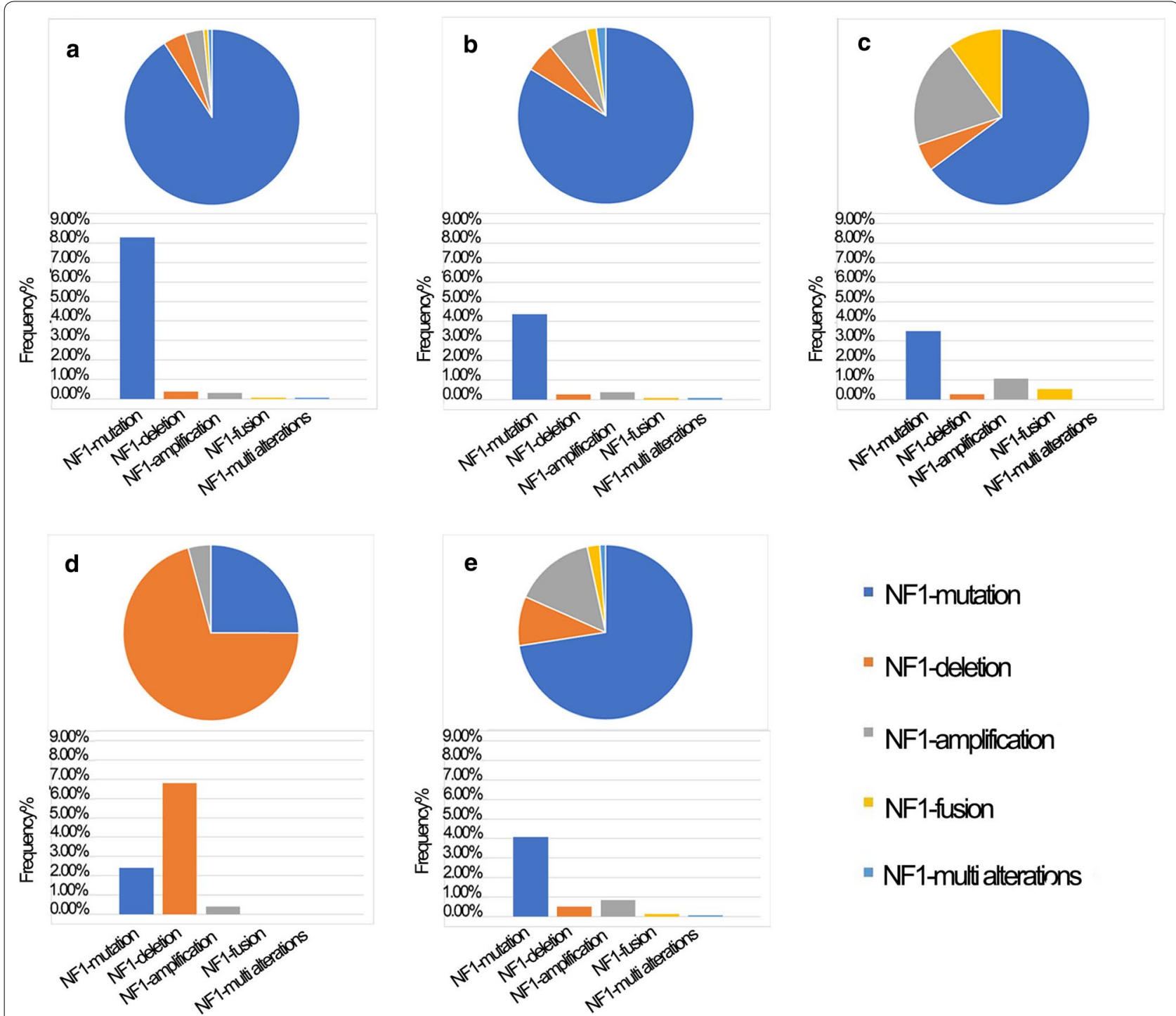

- NF1-mutation

- NF1-deletion

"NF1-amplification

NF1-fusion

- NF1-multialterations

Fig. 1 The types and frequencies of NF1 alterations in different malignancies (data cited from cBioPortal for Cancer Genomics). a NSCLC; b esophagogastric cancer; $\boldsymbol{c}$ liver cancer; $\mathbf{d}$ ovarian cancer; e breast cancer

\section{NF1 gene and neurofibromin}

The NF1 gene was initially discovered as a tumor suppressor in the early 1990s [9-12]. It is located on the long arm 11.2 of chromosome 17 (17q11.2). The types of NF1 mutations are diverse, including missense/nonsense (27.7\%), microdeletions (26.9\%), gross deletions (>20 bp; $13.3 \%)$, splicing (16.3\%), microinsertions (11.1\%), indels (2.0\%), gross insertions (>20 bp; $2.0 \%$ ), complex rearrangements $(0.6 \%)$ and several putative regulatory mutations [13]. Neurofibromin, encoded by the NF1 gene, is a large multi-domain 2818 amino acid protein with a molecular weight of approximately $220 \mathrm{kDa}$ [14]. Neurofibromin contains several domains, including a cysteine-serine-rich domain (CSRD), a central GTPaseactivating protein-related domain (GRD), a tubulin-binding domain (TBD), a SEC14 domain, a carboxy-terminal domain (CTD), a pleckstrin homology (PH) domain and a syndecan-binding domain (SBD). The different domains of the neurofibromin protein is shown in Additional file 1: Figure S1. Neurofibromin binds to GTP-bound Ras through its GRD to regulate the function of Ras. The NF1 gene contains 60 exons and produces multiple alternative splicing isoforms [15]. Exon 23 encodes part of the GRD. An exon 23 splice variant inserts an alternative exon 23a, and exon 23a inclusion specifically decreases the Ras-GAP (GTPase activating protein) activity of 
neurofibromin [16]. Several mechanisms have been confirmed to be associated with the downregulation or even the loss of neurofibromin expression in tumors, such as mutations of the NF1 gene, ubiquitin-mediated proteasomal degradation of neurofibromin [17] and promoter methylation or miRNA-mediated degradation, which inactivates the transcription of the NF1 gene $[18,19]$.

\section{The biological function of the NF1 gene \\ The relationship between NF1 and Ras}

NF1 is a tumor suppressor gene and a negative regulator of Ras protein. Under physiological conditions, neurofibromin, encoded by the NF1 gene, stimulates the GTPase activity of Ras to convert it from an active GTP-bound form to its inactive GDP-bound form through GRD [20]. Therefore, neurofibromin can serve as a shutdown signal for all vertebrate RAS GTPases (including KRAS, NRAS, HRAS, MRAS, RRAS and RRAS2) [21]. The functional loss of neurofibromin caused by NF1 mutations will lead to sustained activation of intracellular RAS-GTP and prolonged activation of the RAS/RAF/MAPK signaling pathway, which eventually results in increased cellular proliferation and even uncontrollable tumor growth. In addition to the loss of the function of the shutdown signal, NF1 mutations increase the number of possible subsequent mutations, which can further upregulate Ras signaling [22]. Ras proteins regulate cell fates by cycling between active GTP-bound and GDP-bound conformations [23] and the activation of the Ras signaling pathway is one of the major driving pathways of malignancies (Table 1).

\section{The relationship between NF1 and other proteins}

Mammalian target of rapamycin (mTOR), an evolutionarily conserved serine-threonine protein kinase, is a downstream effector of Ras and regulates cell proliferation and other biological behaviors [24]. Johannessen et al. demonstrated that neurofibromin was essential for moderately suppressing mTOR signaling in the absence of mitogenic stimuli. They identified NF1 as one of the oncogenes involved in mTOR activation [25]. In mammalian cells, the mTOR complex is a complex with two forms: mTORC1 and mTORC2 [26]. Malone et al. found that mTORC1 was the key PI3K pathway component in malignancies of NF1 mutations, while mTORC2 was dispensable [27]. In addition to Ras and mTORC1, NF1 is associated with various protein molecules, such as focal adhesion kinase (FAK) and valosin-containing protein (VCP). Study by Tsai. et al. reveals NF1 has relationship with integrin/FAK signaling in synapse growth modulation. Genetic and protein-protein interaction between NF1 and FAK suggested that NF1 functions downstream of and forms a protein complex with FAK that mediates NF1 signaling activity and synaptic localization [28]. Wang.et al. showed that neurofibromin and VCP interact and work together to control the density of dendritic spines [29]. These studies suggested neurofibromin, encoded by the NF1 gene, has been shown to regulate synaptogenesis. In this review, we mainly discuss the relationship between NF1 and cancer therapeutic resistance, especially resistance to TKIs; therefore, the relationship between NF1 and other protein molecules will not be restated.

\section{The role of NF1 in anticancer therapies}

Relationship between NF1 mutations and radiotherapy

Studies have shown that radiotherapy increased the incidence of second malignant neoplasms (SMNs) in patients with NF1 mutations [30-32]. Unlike primary cancers, SMNs are therapy-induced malignancies and are becoming a problem that cannot be ignored in cancer survivors.

Choi et al. found that both irradiated wild-type and NF1 mutated mice developed multiple malignancies in a dose-dependent manner in the irradiation field. However, at each radiation dose level, NF1-mutated mice developed more malignancies than matched wild-type mice. They then further analyzed clinical SMN samples and confirmed that among patients with radiation-induced breast cancer, the loss of constitutional heterozygosity $(\mathrm{LOH})$ of $N F 1$ was identified in unrelated individuals without neurofibromatosis type 1 [31]. The mechanism by which NF1 mutations promote radiation-induced

Table 1 The mechanisms of therapeutic resistance induced by NF1 mutations

\begin{tabular}{llll}
\hline Alteration induced by NF1 mutations & Downstream factors & Related therapeutic resistance & References \\
\hline Inhibition of cisplatin-induced apoptosis & MCL1 $\downarrow$ & Cisplatin & [35] \\
Heat shock response & HSF1 $\uparrow$ & Trastuzumab, lapatinib & {$[44-48]$} \\
EMT & Invasion and migration & Targeted therapy & {$[51-54]$} \\
mTOR-HIF-1a-VEGF pathway $\uparrow$ & VEGF $\uparrow$ & TKls & {$[57-60]$} \\
Ras-dependent pathways & Ras $\uparrow$ & TKls & {$[8,41,49]$} \\
\hline
\end{tabular}

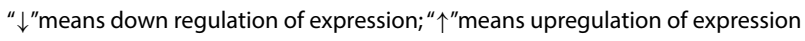


SMNs is poorly understood. Several studies speculated that it might be related to the hyperactivity of the Ras signaling pathway and the loss of tumor protein p53 (TP53) adjacent to NF1 on the chromosome [31, 32], but the specific mechanism needs further research.

\section{The role of NF1 in resistance to chemotherapy and endocrine therapy}

Myeloid cell leukemia 1 (MCL1), which is an antiapoptotic protein in ovarian cancer [33], allows cancer cells to evade apoptosis when its expression is upregulated [34]. Su et al. demonstrated that the loss of NF1 observably upregulated MCL1 expression and endowed ovarian cancer cells with antiapoptotic capability through miR142-5p. They further confirmed that NF1 loss inhibited cisplatin-induced apoptosis and resulted in resistance to chemotherapy in ovarian cancer cells [35].

Sokol et al. demonstrated that NF1 mutations lead to tamoxifen acquired resistance in invasive lobular carcinomas (ILCs) of breast cancer. NF1 mutations are enriched in ILCs, especially in metastatic ILCs, which exhibit a higher frequency of NF1 mutations. Under the appropriate conditions, the loss of NF1 can enhance the competitive growth advantages of breast cancer cells harboring NF1 mutations. Loss of NF1 was detected to cooccur with cadherin 1 (CDH1) inactivation and AKT pathway activation, and either or both of these pathway alterations may facilitate endocrine therapy resistance, which remains to be further studied [36].

\section{The underlying mechanisms of TKI resistance induced by NF1 mutations}

The relationship between NF1 mutations and resistance to TKIs in malignancies has not been extensively explored. Previous studies have demonstrated that mutations in NF1 are related to TKI resistance [8, 20, 37]. However, the mechanism of resistance to TKIs induced by NF1 mutations remains unclear. Nevertheless, research data have suggested that treatments targeting MEK or mTOR are effective and even synergetic for malignancies with NF1 mutations [38-40]. Clinically, mutations of NF1 have also been confirmed to be related to resistance to other agents of targeted therapy. Retinoic acid (RA) is one of the few targeted therapies currently used in the clinic for invasive neuroblastoma. Loss of NF1 contributes to resistance to RA; moreover, the inhibition of MEK signaling downstream restores responsiveness to RA treatment [41]. Another recent clinical study revealed that low expression of NF1 is associated with more extensive lymph node metastases and poor prognosis in epithelial ovarian cancer patients [42]. According to the latest studies, the underlying mechanisms of TKI resistance induced by NF1 mutations are summarized as follows.

\section{Activation of heat shock factor 1}

Heat shock factor 1 (HSF1), an essential conserved master transcriptional regulator in eukaryotic cells, is critical for maintaining homeostasis of the cell proteome [43]. Studies have shown that HSF1 plays an important role in a variety of basic cellular processes essential for carcinogenesis, including cell cycle control, glucose metabolism, ribosome biogenesis, and protein translation. Genetic aberrant HSF1 might attenuate tumorigenesis and cellular transformation driven by oncogene activation or the loss of tumor suppressors both in mice and human cell lines [44, 45]. Dai et al. found that NF1 was a potent regulator of HSF1 and changed the expression and activation of HSF1. NF1 deficiency upregulated HSF1 and activated the heat shock response [46]. A study identified that the augmentation of the HSF1-mediated heat shock response is responsible for lapatinib resistance in breast cancer [47]. Another study showed that increased glycolysis via HSF1 contributes to trastuzumab resistance [48]. Therefore, although there is no direct verification, it is feasible that NF1 mutations might participate in the resistance to TKIs by upregulating HSF1. This mechanism requires further study.

\section{Inhibition of tumor cell apoptosis}

Shapira et al. confirmed that neurofibromin exerted its tumor suppressor function by enhancing the sensitivity of apoptosis via Ras-dependent pathways. In their study, the administration of farnesyl thiosalicylic acid (FTS), which is a Ras inhibitor, increased the apoptosis of neurofibromin-deficient mouse embryonic fibroblasts (MEFs) and malignant peripheral nerve sheath tumor (MPNST) cells [49]. A study provided evidence to suggest that NF1 silencing decreased the sensitivity of erlotinib-induced cell apoptosis and/or growth arrest in lung adenocarcinoma cells. The study revealed that the reduction of neurofibromin expression increased Ras activity and weakened the effect of erlotinib on the downstream MAPK pathway, thereby decreasing the sensitivity of EGFR inhibitory drugs and eventually leading to the resultant resistance to erlotinib. Neurofibromin influenced erlotinib sensitivity through its function as a negative regulator of Ras protein [8]. Therefore, the antiapoptotic effect caused by NF1 mutations through the Ras-dependent pathway may be one of the mechanisms of TKI resistance in malignancies.

\section{Promotion of epithelial-mesenchymal transformation}

Epithelial-mesenchymal transition (EMT) is a process of the loss of epithelial characteristics and the acquisition 
of a mesenchymal phenotype, which is mediated by the activation of EMT transcription factors (EMT-TFs) [50]. The EMT process is associated with the resistance of multiple therapeutics in tumor cells by enhancing the migration and invasion of tumor cells. Tumor cells harboring the EMT phenotype showed intrinsic resistance to EGFR TKIs [51-53]. Arima et al. demonstrated that silencing NF1 induced the expression of EMT-TFs in normal human Schwann cells and epithelioid breast cancer cells, suggesting that the loss of neurofibromin expression might activate the EMT-related signaling pathway [54]. As a result, neurofibromin might inhibit the EMT process, while NF1 mutations, which contribute to the loss of neurofibromin expression, could be the underlying mechanism of TKI resistance in malignancies.

\section{Promotion of sustained angiogenesis}

Angiogenesis is necessary for tumor growth and metastasis, and the transition to an angiogenic phenotype depends on the result of a balance between pro-angiogenic and anti-angiogenic factor expression for most tumors [55]. Using NF1 heterozygous mice model, Wu et al. demonstrated increased neovascularization in both the retina and cornea in response to hypoxia and bFGF, which was associated with heightened endothelial cell proliferation and migration, and increased infiltration of inflammatory cells including macrophage and mast cells [56]. Thomas et al. found that compared to normal human Schwann cells, neurofibromin deficiency was associated with the upregulation of proangiogenic factors and the downregulation of antiangiogenic factors, which enhanced the carcinogenicity of carcinogenic Schwann cells [57]. Kawachi et al. demonstrated that NF1 gene silencing in both Schwann cells and non-Schwann cells directly leads to activation of the mTOR- hypoxia-inducible factor- $1 \alpha$ (HIF-1 $\alpha)$ - vascular endothelial growth factor (VEGF) pathway, which in turn increases VEGF expression [58]. Bevacizumab, a well-known VEGF inhibitor, inhibits tumor proliferation and angiogenesis through the inhibition of the VEGF pathway. A study showed that after treatment with bevacizumab, adult recurrent high-grade glioma patients harboring NF1 mutations had prolonged postrecurrence survival [59]. Angiogenesis was proven to be related to TKI resistance [60], and NF1 mutations might participate in the resistance to TKIs by promoting tumor angiogenesis. However, this mechanism has not been directly verified, and further research is needed. The role of NF1 mutations in chemotherapy and TKI resistance is shown in Fig. 2.

Based on the findings above, NF1 mutations might directly or indirectly lead to changes in several important biological behaviors of malignancies, including cell proliferation, antiapoptosis, angiogenesis, and metastasis. These behaviors are related to not only resistance to targeted therapy but also other anticancer therapeutics. This suggests that NF1 mutations play an important role in the generation and development of malignancies and might induce resistance to anticancer therapy. The mechanisms of therapeutic resistance induced by NF1 mutations are also summarized in Table 1.

\section{Novel therapies for NF1 mutant malignancies}

As a negative regulator of Ras signaling, NF1 loss results in Ras-dependent drug resistance. Previous studies have shown that the application of inhibitors of Ras and its downstream targets could overcome drug resistance induced by NF1 mutations. Ras inhibitors, such as FTS, and mTOR inhibitors, such as everolimus, can inhibit the growth of NF1 mutated malignancies [61, 62]. Beauchamp et al. found that for NF1-mutated lung adenocarcinoma resistant to dasatinib, the knockdown of ERK1/2 was sufficient to kill dasatinib-resistant cells [37]. Treatment of lung cancer with low levels of NF1 expression with MAP-ERK kinase (MEK) inhibitors can restore sensitivity to erlotinib and reverse erlotinib resistance. Therefore, concurrently using EGFR and MEK inhibitors might be superior to monotherapy for TKI-resistant NF1-mutated lung cancer [8]. In other studies, inhibitors of PI3K, the downstream effector of the Ras pathway, were shown to inhibit the growth of NF1-mutated MPNST cells and neurofibromin-deficient human breast cancer xenografts in mice $[63,64]$. STAT3 is a downstream molecule of the PI3K/Akt/mTOR pathway, and natural cucurbitacin-I is a potent STAT3 inhibitor that inhibits the growth of NF1-mutated MPNST cells in vitro and in vivo [65].

Other therapies for NF1 mutated malignancies, such as anti-angiogenic drugs and HSF1 inhibitors, have been reported. However, further research is still needed [46, $58,66]$.

\section{Conclusion}

In conclusion, drug resistance is a serious problem in the clinical treatment of malignancies. Understanding the mechanisms of drug resistance is critical for the development of new therapeutic strategies. According to our review, NF1 mutations play an important role in the generation, development and drug resistance of malignancies. NF1 mutations are involved in chemotherapy and targeted therapy resistance in tumor cells through multiple mechanisms. Inhibition of NF1 downstream targets is an effective strategy for overcoming resistance induced by NF1 mutations. Therefore, NF1 


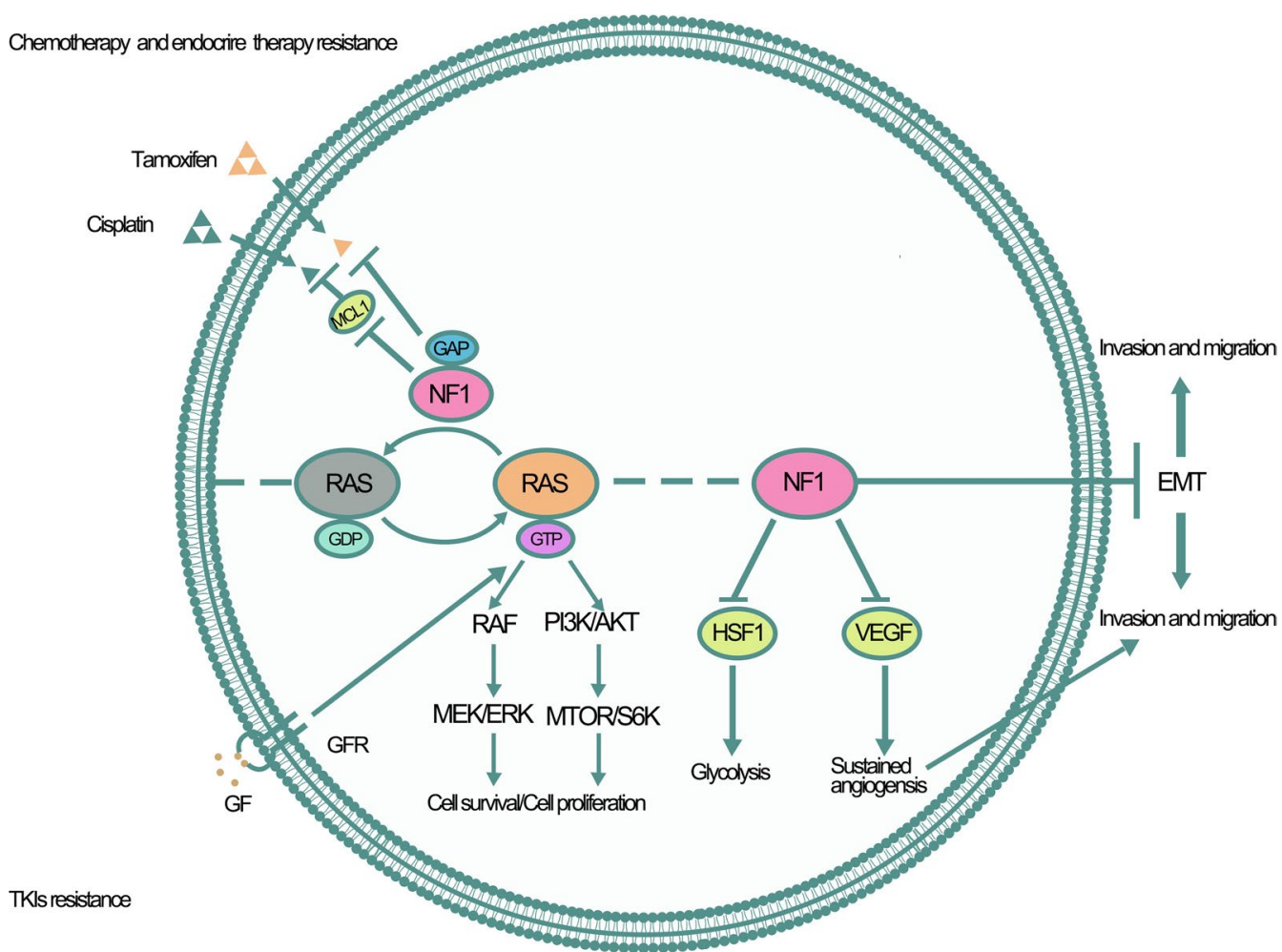

Fig. 2 The role of NF1 in resistance to anticancer therapeutics. NF1 might be involved in resistance to anticancer therapeutics through several mechanisms, which included: NF1 downregulated MCL1 expression and endowed cells with cisplatin resistance; NF1 mutations leaded to tamoxifen acquired resistance and the mechanism was still uncertain; NF1 mutations participated in TKIs resistance via promoting glycolysis, angiogenesis, EMT, cellular proliferation and survival. GTP=guanosine triphosphate. RAS = rat sarcoma viral oncogene homologue. $\mathrm{GDP}=$ guanosine diphosphate. RAF = murine sarcoma viral oncogene homologue. MEK=MAPK-ERK kinase. PI3K= phosphatidylinositol-3-kinase. $A K T=V$-akt murine thymoma viral oncogene homologue 1. $\mathrm{mTOR}=$ mammalian target of rapamycin. Rac1 = Ras-related C3 botulinum toxin substrate 1. PAK1 $=$ P21-Activated Kinase

mutations might be novel therapeutic targets for cancer treatment.

\section{Supplementary information}

Supplementary information accompanies this paper at https://doi. org/10.1186/s12935-020-01570-8.

Additional file 1: Figure S1. The different domains of the neurofibromin protein.

\section{Abbreviations}

NF1: Neurofibromin 1; EGFR: Epidermal growth factor receptor; EGF: Epidermal growth factor; TKI: Tyrosine kinase inhibitor; EMT: Epithelial-mesenchymal transition; HSF1: heat shock factor 1; MCL1: Myeloid cell leukemia 1; GRD: GTPaseactivating protein-related domain; VEGF: Vascular endothelial growth factor.

\section{Acknowledgements}

We acknowledge Tao Wang (Huaiyin Normal University) for his kind guidance in making pictures.

\section{Authors' contributions}

JT Conceptualization and writing the original draft; DS and LD Data collection and writing of the original draft; $\mathrm{HH}$ Conceptualization. All authors reviewed the manuscript. All authors read and approved the final manuscript.

\section{Funding}

This work was supported by Key Research and Development Project of Shandong Province (2017GSF18147 to Helei Hou) and Special Funding for Qilu Sanitation and Health Leading Talents Cultivation Project (to Helei Hou).

\section{Availability of data and materials}

The data and material in this review all come from published papers.

\section{Ethics approval and consent to participate}

Not applicable.

\section{Consent to publication}

Not applicable.

\section{Competing interests}

The authors declare that they have no competing interests.

Received: 16 July 2020 Accepted: 23 September 2020

Published online: 09 October 2020 


\section{References}

1. Ferlay J, et al. Estimating the global cancer incidence and mortality in 2018: GLOBOCAN sources and methods. Int J Cancer. 2019;144(8):1941-53.

2. Ma WW, Adjei AA. Novel agents on the horizon for cancer therapy. CA Cancer J Clin. 2009;59(2):111-37.

3. Ramalingam SS, Owonikoko TK, Khuri FR. Lung cancer: New biological insights and recent therapeutic advances. CA Cancer J Clin 2011;61(2):91-112.

4. Vogel CL, et al. Efficacy and safety of trastuzumab as a single agent in first-line treatment of HER2-overexpressing metastatic breast cancer. J Clin Oncol. 2002;20(3):719-26.

5. Glickman MS, Sawyers CL. Converting cancer therapies into cures: lessons from infectious diseases. Cell. 2012;148(6):1089-98.

6. Ferner RE. Neurofibromatosis 1 and neurofibromatosis 2: a twenty first century perspective. Lancet Neurol. 2007:6(4):340-51.

7. Maertens $\mathrm{O}$, et al. Elucidating distinct roles for NF1 in melanomagenesis Cancer Discov. 2013:3(3):338-49.

8. de Bruin EC, et al. Reduced NF1 expression confers resistance to EGFR inhibition in lung cancer. Cancer Discov. 2014;4(5):606-19.

9. Cawthon RM, et al. A major segment of the neurofibromatosis type 1 gene: cDNA sequence, genomic structure, and point mutations. Cell. 1990;62(1):193-201.

10. Downward J. Plugging the GAPs. Curr Biol. 1991;1(6):353-5.

11. Viskochil $D$, et al. Deletions and a translocation interrupt a cloned gene at the neurofibromatosis type 1 locus. Cell. 1990;62(1):187-92.

12. Wallace $M R$, et al. Type 1 neurofibromatosis gene: identification of a large transcript disrupted in three NF1 patients. Science. 1990;249(4965):181-6.

13. Stenson PD, et al. The Human Gene Mutation Database: towards a comprehensive repository of inherited mutation data for medical research, genetic diagnosis and next-generation sequencing studies. Hum Genet. 2017;136(6):665-77.

14. Daston MM, et al. The protein product of the neurofibromatosis type 1 gene is expressed at highest abundance in neurons, Schwann cells, and oligodendrocytes. Neuron. 1992;8(3):415-28.

15. Shen MH, Harper PS, Upadhyaya M. Molecular genetics of neurofibromatosis type 1 (NF1). J Med Genet. 1996;33(1):2-17.

16. Hinman $M N$, et al. Neurofibromatosis type 1 alternative splicing is a key regulator of Ras signaling in neurons. Mol Cell Biol. 2014;34(12):2188-97.

17. Hollstein PE, Cichowski K. Identifying the Ubiquitin Ligase complex that regulates the NF1 tumor suppressor and Ras. Cancer Discov. 2013:3(8):880-93.

18. Gutmann DH, et al. Somatic neurofibromatosis type 1 (NF1) inactivation characterizes NF1-associated pilocytic astrocytoma. Genome Res. 2013;23(3):431-9.

19. Lenarduzzi M, et al. MicroRNA-193b enhances tumor progression via down regulation of neurofibromin 1. PLOS ONE. 2013;8(1):e53765.

20. Whittaker SR, et al. A genome-scale RNA interference screen implicates NF1 loss in resistance to RAF inhibition. Cancer Discov. 2013:3(3):350-62.

21. Ohba Y, et al. Regulatory proteins of R-Ras, TC21/R-Ras2, and M-Ras/RRas3. J Biol Chem. 2000;275(26):20020-6.

22. Stites EC, et al. Cooperation between Noncanonical Ras Network Mutations. Cell Rep. 2015;10(5):840.

23. Vetter IR, Wittinghofer $A$. The guanine nucleotide-binding switch in three dimensions. Science. 2001:294(5545):1299-304.

24. Hay N, Sonenberg N. Upstream and downstream of mTOR. Genes Dev. 2004;18(16):1926-45.

25. Johannessen CM, et al. The NF1 tumor suppressor critically regulates TSC2 and mTOR. Proc Natl Acad Sci U S A. 2005;102(24):8573-8.

26. Sabatini DM. mTOR and cancer: insights into a complex relationship. Nat Rev Cancer. 2006;6(9):729-34

27. Malone $\mathrm{CF}_{\text {, et }}$ al. Defining key signaling nodes and therapeutic biomarkers in NF1-mutant cancers. Cancer Discov. 2014;4(9):1062-73.

28. Tsai PI, et al. Neurofibromin mediates FAK signaling in confining synapse growth at Drosophila neuromuscular junctions. J Neurosci. 2012;32(47):16971-81.

29. Wang HF, et al. Valosin-containing protein and neurofibromin interact to regulate dendritic spine density. J Clin Invest. 2011;121(12):4820-37.

30. Chao RC, et al. Therapy-induced malignant neoplasms in $\mathrm{Nf1}$ mutant mice. Cancer Cell. 2005;8(4):337-48.
31. Choi G, et al. Genetically mediated Nf1 loss in mice promotes diverse radiation-induced tumors modeling second malignant neoplasms. Cancer Res. 2012:72(24):6425-34.

32. Nakamura $\mathrm{J}$, et al. Dose-dependent effects of focal fractionated irradiation on secondary malignant neoplasms in Nf1 mutant mice. Cancer Res. 2011;71(1):106-15.

33. Wertz IE, et al. Sensitivity to antitubulin chemotherapeutics is regulated by MCL1 and FBW7. Nature. 2011;471(7336):110-4.

34. Ertel F, et al. Programming cancer cells for high expression levels of $\mathrm{Mcl} 1$. EMBO Rep. 2013;14(4):328-36.

35. Su J, et al. NF1 regulates apoptosis in ovarian cancer cells by targeting MCL1 via miR-142-5p. Pharmacogenomics. 2019;20(3):155-65.

36. Sokol ES, et al. Loss of function of NF1 is a mechanism of acquired resistance to endocrine therapy in lobular breast cancer. Ann Oncol. 2019;30(1):115-23.

37. Beauchamp EM, et al. Acquired resistance to dasatinib in lung cancer cell lines conferred by DDR2 gatekeeper mutation and NF1 loss. Mol Cancer Ther. 2014:13(2):475-82.

38. Johannessen CM, et al. TORC1 is essential for NF1-associated malignancies. Curr Biol. 2008;18(1):56-62.

39. Jousma E, et al. Preclinical assessments of the MEK inhibitor PD-0325901 in a mouse model of Neurofibromatosis type 1. Pediatr Blood Cancer. 2015;62(10):1709-16

40. Varin J, et al. Dual mTORC1/2 inhibition induces anti-proliferative effect in NF1-associated plexiform neurofibroma and malignant peripheral nerve sheath tumor cells. Oncotarget. 2016;7(24):35753-67.

41. Holzel M, et al. NF1 is a tumor suppressor in neuroblastoma that determines retinoic acid response and disease outcome. Cell. 2010;142(2):218-29.

42. Qiao G, et al. Neurofibromin 1 expression is negatively correlated with malignancy and prognosis of epithelial ovarian cancer. Int J Clin Exp Pathol. 2019;12(5):1702-12.

43. Balch $W E$, et al. Adapting proteostasis for disease intervention. Science. 2008;319(5865):916-9.

44. Mendillo ML, et al. HSF1 drives a transcriptional program distinct from heat shock to support highly malignant human cancers. Cell. 2012;150(3):549-62.

45. Dai $C$, et al. Heat shock factor 1 is a powerful multifaceted modifier of carcinogenesis. Cell. 2007;130(6):1005-18.

46. Dai C, et al. Loss of tumor suppressor NF1 activates HSF1 to promote carcinogenesis. J Clin Invest. 2012;122(10):3742-54

47. Yallowitz $A$, et al. Heat shock factor 1 confers resistance to lapatinib in ERBB2-positive breast cancer cells. Cell Death Dis. 2018;9(6):621.

48. Zhao $Y$, et al. Overcoming trastuzumab resistance in breast cancer by targeting dysregulated glucose metabolism. Cancer Res. 2011;71(13):4585-97.

49. Shapira S, et al. The tumor suppressor neurofibromin confers sensitivity to apoptosis by Ras-dependent and Ras-independent pathways. Cell Death Differ. 2007:14(5):895-906.

50. Thiery JP, et al. Epithelial-mesenchymal transitions in development and disease. Cell. 2009:139(5):871-90.

51. Frederick BA, et al. Epithelial to mesenchymal transition predicts gefitinib resistance in cell lines of head and neck squamous cell carcinoma and non-small cell lung carcinoma. Mol Cancer Ther. 2007;6(6):1683-91.

52. Rho JK, et al. Epithelial to mesenchymal transition derived from repeated exposure to gefitinib determines the sensitivity to EGFR inhibitors in A549, a non-small cell lung cancer cell line. Lung Cancer. 2009:63(2):219-26.

53. Thomson S, et al. Epithelial to mesenchymal transition is a determinant of sensitivity of non-small-cell lung carcinoma cell lines and xenografts to epidermal growth factor receptor inhibition. Cancer Res. 2005:65(20):9455-62.

54. Arima Y, et al. Decreased expression of neurofibromin contributes to epithelial-mesenchymal transition in neurofibromatosis type 1. Exp Dermatol. 2010;19(8):e136-41.

55. Folkman J. The role of angiogenesis in tumor growth. Semin Cancer Biol. 1992;3(2):65-71.

56. Wu M, Wallace MR, Muir D. Nf1 haploinsufficiency augments angiogenesis. Oncogene. 2006;25(16):2297-303. 
57. Thomas SL, De Vries GH. Angiogenic expression profile of normal and neurofibromin-deficient human Schwann cells. Neurochem Res. 2007;32(7):1129-41.

58. Kawachi Y, et al. NF1 gene silencing induces upregulation of vascular endothelial growth factor expression in both Schwann and nonSchwann cells. Exp Dermatol. 2013;22(4):262-5.

59. Theeler BJ, et al. Prolonged survival in adult neurofibromatosis type I patients with recurrent high-grade gliomas treated with bevacizumab. $J$ Neurol. 2014;261(8):1559-64.

60. Kerbel RS. Inhibition of tumor angiogenesis as a strategy to circumvent acquired resistance to anti-cancer therapeutic agents. BioEssays. 1991;13(1):31-6.

61. Barkan B, et al. The Ras inhibitor farnesylthiosalicylic acid as a potential therapy for neurofibromatosis type 1. Clin Cancer Res. 2006;12(18):5533-42.

62. Endo $M$, et al. Prognostic significance of AKT/mTOR and MAPK pathways and antitumor effect of mTOR inhibitor in NF1-related and sporadic malignant peripheral nerve sheath tumors. Clin Cancer Res. 2013;19(2):450-61.
63. Hirokawa Y, et al. Signal therapy of human pancreatic cancer and NF1-deficient breast cancer xenograft in mice by a combination of PP1 and GL-2003, anti-PAK1 drugs (Tyr-kinase inhibitors). Cancer Lett. 2007;245(1-2):242-51.

64. Hirokawa Y, et al. Sichuan pepper extracts block the PAK1/cyclin D1 pathway and the growth of NF1-deficient cancer xenograft in mice. Cancer Biol Ther. 2006;5(3):305-9.

65. Banerjee $\mathrm{S}$, et al. The neurofibromatosis type 1 tumor suppressor controls cell growth by regulating signal transducer and activator of transcription-3 activity in vitro and in vivo. Cancer Res. 2010;70(4):1356-66.

66. De Raedt T, et al. Exploiting cancer cell vulnerabilities to develop a combination therapy for ras-driven tumors. Cancer Cell. 2011;20(3):400-13.

\section{Publisher's Note}

Springer Nature remains neutral with regard to jurisdictional claims in published maps and institutional affiliations.
Ready to submit your research? Choose BMC and benefit from:

- fast, convenient online submission

- thorough peer review by experienced researchers in your field

- rapid publication on acceptance

- support for research data, including large and complex data types

- gold Open Access which fosters wider collaboration and increased citations

- maximum visibility for your research: over $100 \mathrm{M}$ website views per year

At BMC, research is always in progress.

Learn more biomedcentral.com/submissions 